\title{
Technophobia, prescription checking and the future of diabetes management
}

\author{
A. M. Albisser \\ Received: 4 February 2009 /Accepted: 23 February 2009 / Published online: 9 April 2009 \\ (C) Springer-Verlag 2009
}

\begin{abstract}
Is the medical prescription the root cause of the long-term complications of diabetes mellitus? This article presents the argument for introducing prescription checking into diabetes disease management. It discusses an evidencebased need for frequent revisions of the medical prescription as the key to preventing treatment-related complications in diabetes while achieving the now mandated standards in patient care. To do this it will be prudent for diabetes healthcare providers to enrich their clinical services with new information technology-based tools. These are easily acquired by participating in professional workshops focused on advanced diabetes management. In this light, the case presented here challenges leading practitioners to become early participants in the evolution of information technology that will ultimately enhance the management of all patients with diabetes.
\end{abstract}

Keywords Diabetes complications · Glucose control · Hypoglycaemia $\cdot$ Information technology $\cdot$ Medical practice $\cdot$ Therapy

\section{Abbreviation \\ SMBG Self-monitored blood glucose}

A. M. Albisser $(\bowtie)$

Diabetes Control and Complications Treatment Initiative,

BCMC Better Control Medical Computers,

Hollywood, FL, USA

e-mail: albisser@nidm.org

A. M. Albisser

DCCT Initiative,

1400 South Ocean Drive, Suite 604,

Hollywood, FL 33019, USA
Fragmented resources in diabetes management

Are there resources available to doctors to help them reach the goals of glycaemic control and body weight now mandated by the professional societies? Or is this the sole responsibility of the diabetes educator? A participant in our local diabetes support group professed that her diabetes education sessions were frustrating. She explained that it was like having a talented personal trainer teach her to ride a bicycle: the educator appointed was an expert rider and, for safety, a tandem bicycle was used in teaching the skills of riding. When the sessions ended, however, the educator not only left the scene but also took the bicycle away! Feeling abandoned in this way after educational sessions is not uncommon.

Is it wise and prudent to deny healthcare providers and their patients the proper tools to carry out the tasks involved in the management of diabetes? Think of how much home blood glucose monitoring is promoted and (painfully) performed each day yet how little any of the measurements are actually used. Is this not an awful waste? As currently practised, self-monitored blood glucose (SMBG) readings generally fail to represent the patient's actual metabolic profile in a meaningful way. However, they could, and if they did, SMBG would be invaluable. For this reason alone, is it not time to consider diabetes to be not just a metabolic disorder, but also a systems problem with an urgent need to close what is now a fragmented circle of care?

\section{Evidence-based need for a frequent review of prescriptions}

In light of the evidence afforded by the DCCT [1] and the UK Prospective Diabetes Study [2], the medical prescrip- 
tion for the control of glycaemia has become central to patient management. Convinced by the trials' remarkable results, and pushed to action by the widespread media coverage, care providers everywhere moved to embrace the concepts of intensified therapy as originally defined in the trial protocols. With great effort and substantially increased costs, they prescribed intensified therapies for their patients, including the provision of education in greater detail, diets featuring carbohydrate counting and pharmacotherapy involving multiple daily injections, continuously pumped insulin or combination therapy. Gratifying improvements were anxiously awaited.

\section{So, what went wrong?}

Contrary to expectations, the outcomes of intensified therapy in clinical practice were disappointing in terms of glycaemic control and $\mathrm{HbA}_{1 \mathrm{c}}$. Furthermore, the consequent rise in iatrogenic hypoglycaemia [3] has been epidemic but tolerated, as unfortunately it is believed to be the only way to achieve the mandated glycaemic targets [4]. Is this really a case of no pain, no gain?

\section{Why has this happened? The protocol 'patch' that made intensified therapy work}

With hindsight, it appears that the reporting of the striking clinical results of the landmark DCCT under-emphasised crucial methodological details. It was widely overlooked that, while just beginning to implement the original trial protocol, the investigators realised that their prescriptions for intensified therapy per se were insufficient to meet the tight glycaemic goals required in the experimental group.

To remedy this, the clinical prescriptions were urgently expanded to include increased patient-provider contact. This patch to the protocol was accomplished by increased monitoring. In effect, intensified therapy as then practised throughout the DCCT included weekly home visits with frequent telephone contacts in between. This was done manually by one or more team members, including study nurses, diabetes nurse educators and/or caseworkers.

At each repeated encounter, one or more of the team members worked diligently to review crucial outcomes in light of the current medical prescription. Then, as deemed necessary at that particular time, diabetes education was reinforced and/or immediate changes were made in the medical prescription. Corrections, especially in dosing rates, were frequent, cautiously avoiding hypoglycaemia, with care taken not to compromise overall glycaemia and unfavourably affect subsequent $\mathrm{HbA}_{1 \mathrm{c}}$ values. Parenthetically, the goal of both trials was to realise lower $\mathrm{HbA}_{1 \mathrm{c}}$ values in the experimental (intensified therapy) subgroups compared with the control (standard therapy) subgroups.

\section{What about the patch that worked?}

In the rush to bring the heralded promises of these two trials to the benefit of their patients, practitioners everywhere tended to overlook the overarching need to update the therapy for each patient on a weekly basis. If such intensive support and frequent prescription corrections had been recognised as essential, few practitioners would have transferred their patients to intensified therapies. In short, even if the necessary skills are not lacking, the intensified support required is simply impractical, in terms of prolonged contact time and reimbursement. The medical encounter for diabetes management is already complicated enough without requiring a patch that further increases time and effort.

\section{The clinical encounter and the diabetes prescription review}

The systems-like flow diagram in Fig. 1 shows that patients on diabetes therapy should each present an array of data to the attending healthcare provider during each encounter. When any item in the array is outside its specific target range, the provider must intervene, which usually involves modifying the prescription in such a way as to resolve the deviation.

Only when such 'prescription corrections' (the patch to the DCCT protocol) were performed weekly, or more often, did metabolic control consistently meet desired target values. Evidently, when the frequency of such prescription review is relaxed to monthly or quarterly intervals in clinical practice, metabolic control is unlikely to improve [3] and, in fact, often degrades.

\section{So, what is missing? Turning the DCCT patch into a tool}

The DCCT participants in the intervention group showed significant reductions in the rates of diabetes-related complications. Sadly, many of these benefits were evanescent, gradually disappearing once the frequent 'prescription corrections' were terminated. This convergence of the experimental group with the poor outcomes expected in the control group occurred in spite of continuing the prescribed intensified therapies beyond the conclusion of the trials. Notably, $\mathrm{HbA}_{1 \mathrm{c}}$ has been determined to explain virtually all of the difference in the risk of complications between the intensive and conventional groups [4]. 
Fig. 1 The current model. At routine face-to-face encounters the patient on diabetes therapy presents key outcomes to the attending provider for review. Decisions are made to revise the medical prescription when targets are not met, including SMBG values, body weight (BW), blood pressure, lipids, medication types, medication dosages, carbohydrate counts (CHO), exercise, stress. Rx, treatment

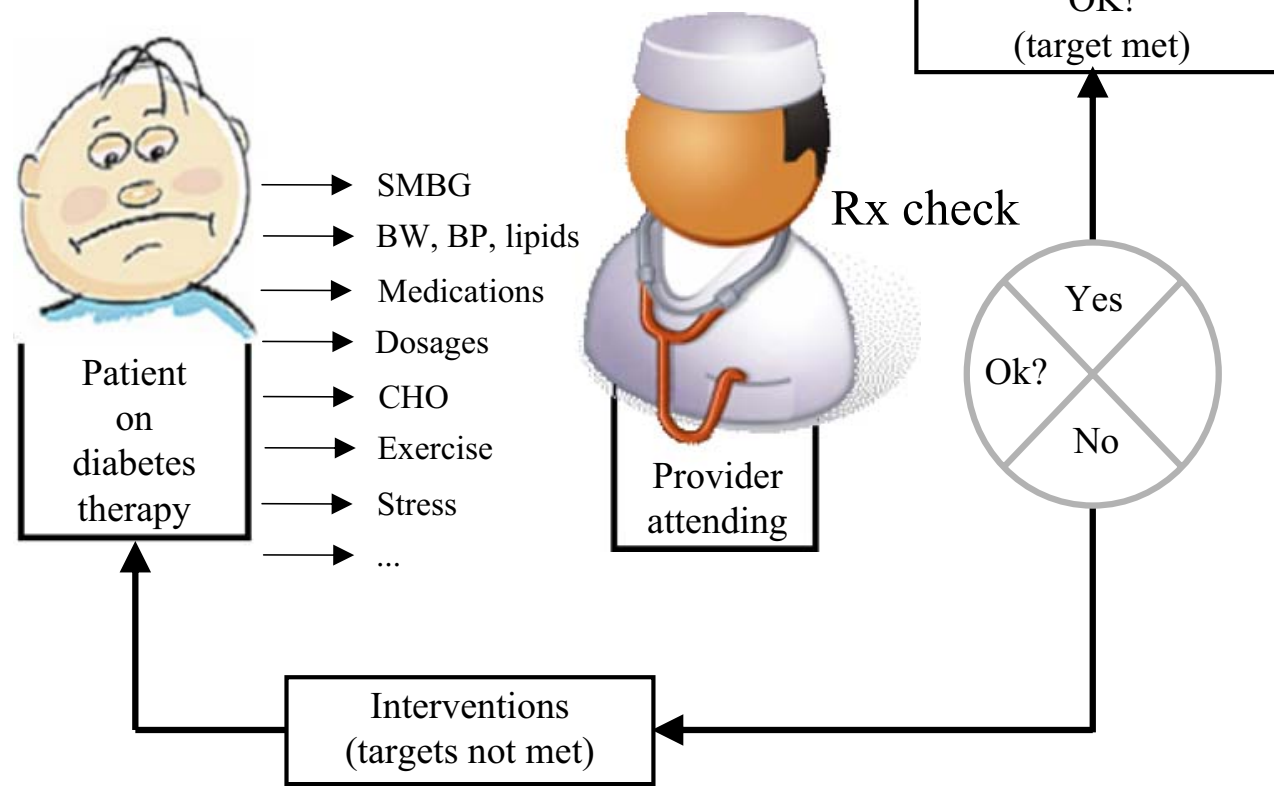

Control of glycaemia is imperative. Evidently, the DCCT patch is essential. Without the frequent 'prescription corrections' of the trials, either metabolic control deteriorates or adverse side effects dominate $[5,6]$.

Arguably, a tool is needed that patches a 'virtual' encounter in place of a 'real' encounter. The tool is to support a limited prescription check for each patient at requisite intervals. The list of items that could be tracked by such a tool includes SMBG, body weight, blood pressure, medication types, dosing, carbohydrates, exercise, stress and performance. As shown in Fig. 2, information technology must patch for the remote provider and monitor key outcomes to identify when real contact with the provider is needed.

\section{Properties of a new tool to replace the DCCT patch}

Technologically, what properties should a prescriptionchecking tool possess? The preliminary list includes only
Fig. 2 A new model. Information technology that includes a database and supporting software allows virtual encounters to substitute for the remote provider. Prescription checks are realised by monitoring key outcomes. Whenever the new tool detects that targets are unmet, including SMBG values, body weight (BW) and carbohydrate counts $(\mathrm{CHO})$, it calls for provider contact and thereby triggers timely corrective interventions. Rx, treatment

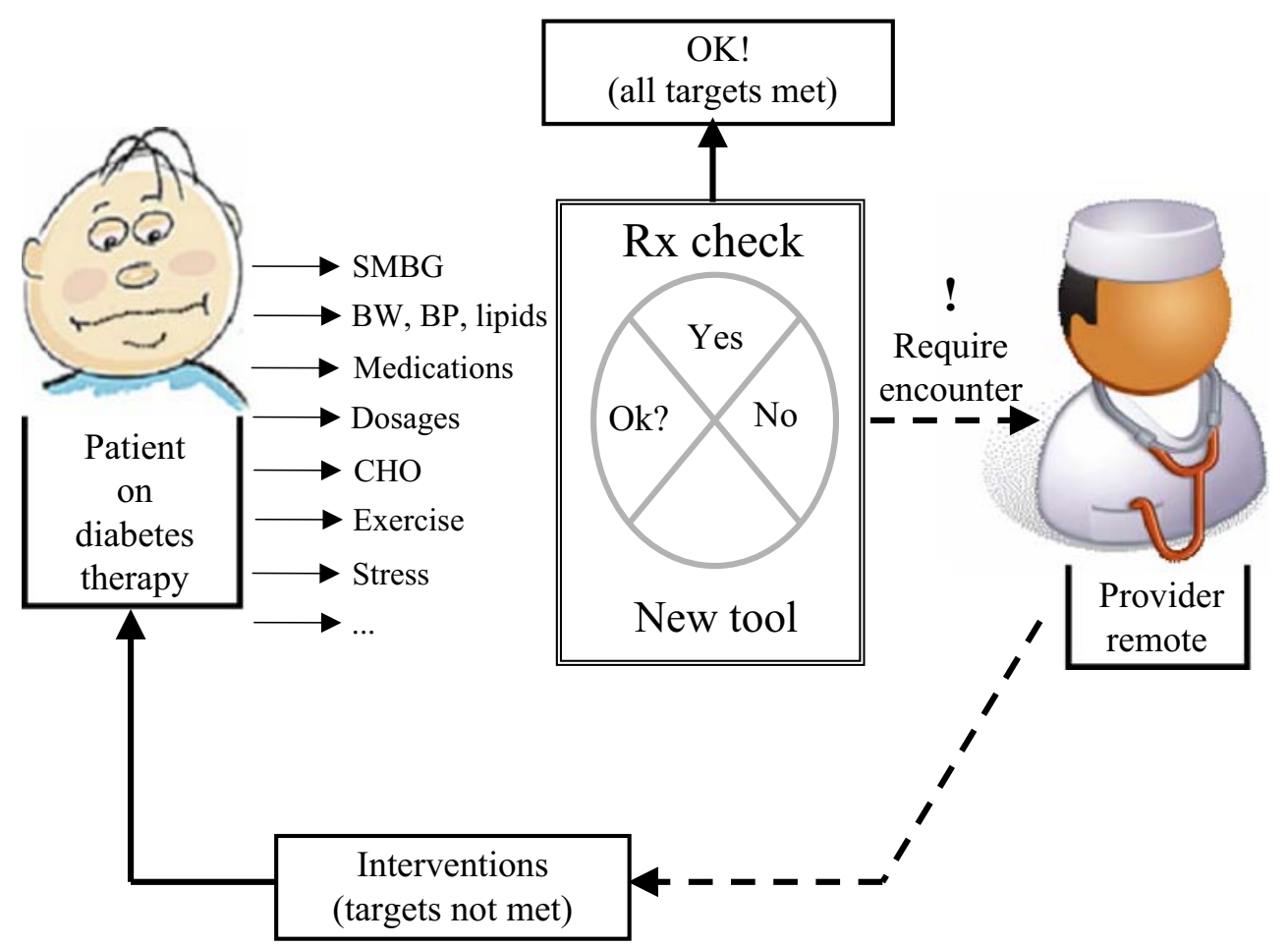


those that contemporary information technology can support. Without doubt, such a tool should have the following properties.

1. It should be simple, user-friendly, portable, robust and secure.

2. With even modest digital memory, it should contain a life-long, personal diabetes database that includes patient and provider registries, and serve as a mini electronic medical record for diabetes.

3. It should contain software to execute a variety of calculations or statistical analyses on the cumulating data that are stored internally.

4. Such procedures should include the display of the actual glycaemic profiles, statistical analyses, trend determinations and even simulations to predict outcomes of possible interventions.

5. With ongoing timely data entries by the patient at home, such a tool should offer 'virtual' provider contacts and thereby realise the needed prescription checks at requisite intervals.

6. It should complement the handwritten diabetes diary.

7. It should facilitate tracking the performance of the provider (and the patient) in meeting mandated outcomes.

In short, where poor outcomes with intensified therapies are caused by prescriptions that have drifted out of date or that are simply wrong, a new tool is needed. It must substitute for the weekly patient-provider contacts and prescription reviews that proved fundamental to the success of the DCCT and UKDPS.

In clinical practice, will such prescription checks also prove fundamental to achieving and maintaining metabolic control that will reduce long-term diabetes-related complications while mitigating iatrogenic hypoglycaemia, pharmacological over-treatment, insulin resistance and body weight gain? Based on the available evidence, they certainly will.

One has to wonder if there is any reason why userfriendly information technology has not already substituted for the DCCT patch? If we can put a man on the moon and return him safely, why have we not met this comparatively simple practice need?

\section{A new DCCT initiative?}

Recognising the potential value of repeated prescription checks and the need to do these by virtual rather than real patient-provider contacts, my colleagues and I at Better Control Medical Computers are looking to form a collaboration called the DCCT initiative. The acronym stands for the Diabetes Control and Complications Treatment initiative, borrowing from the original National Institutes of
Health study entitled the Diabetes Control and Complications Trial. In effect, the goal is to realise the translation of trial methodology into patient treatment.

The new DCCT initiative is mainly an educational undertaking. The plan is to offer short (1.5 day) workshops for providers and technologists interested in bringing the tools of information technology (decision support and prescription checking) into clinical practice, particularly for the benefit of those patients whose diabetes is proving difficult to manage.

The initiative is intended to support the management of both type 1 and type 2 diabetes mellitus and includes all contemporary information technologies that are ready for clinical implementation.

\section{Drug and device qualification}

The development of information technology is different from that of medical devices and drugs. Drugs cannot be altered in the field. To a limited extent, devices can be updated in the field but are usually recalled for modification or replacement. In contrast, information technology can be readily updated. Computer programs, being essentially works in progress, can be rapidly corrected while in service, which allows continuing improvements and crucial updates to be made so that all the needs of each user can be met. Think of the evolution of open-source code. Even changing requirements can be accommodated. Thus, with very short development times, collaborations can drive device qualification and lead to products that truly meet everyone's needs. The goal of the DCCT initiative is to facilitate this.

\section{Growth in decision support}

A search of PubMed with the keywords 'diabetes' AND 'decision support' reveals 306 articles over the period 1985 to 2008. As shown in Fig. 3, growth in the number of papers on this subject published in technical and clinical journals has been accelerating. A move to prescription checking in diabetes is just beginning [7, 8]. Many of the above publications confirm that decision support technologies do improve outcomes in diabetes.

\section{Technophobia}

In recent times, information technology has been embraced to a remarkable degree by many medical disciplines, including radiography, neurology and cardiology. Over the years there have been significant attempts to develop tools 


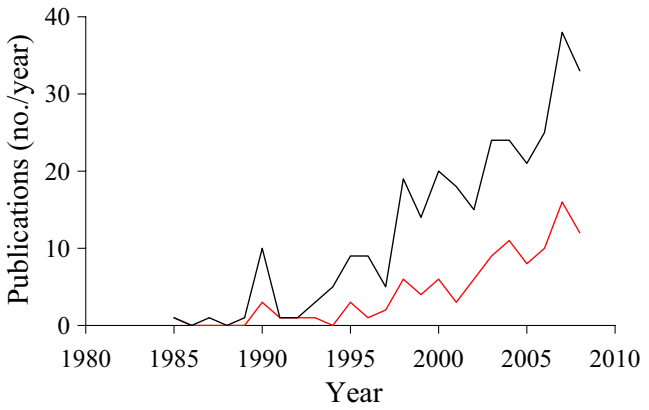

Fig. 3 The rise in publications featuring decision support in diabetes over the last 20 years. The red curve shows publications in clinical journals; the black curve adds publications in technical journals

for diabetes disease management, clinical decision support and patient self-management. Curiously, these innovations seem to have been marginalised by diabetes practitioners. 'I will never allow a computer to manage a single dose of my patients' medications' has been a prevalent and persistent attitude. As are the statements 'Candidly, I am afraid of computers and so are my patients', 'My patients do exactly, I repeat, exactly, what I instruct them to do. They have no need to call in their results to remote monitoring services', and 'My patients have brains and they should use them'. Surprisingly, these attitudes are rarely due to adverse experiences with any flawed patient care technologies.

Is this simply technophobia? Perhaps it just stems from a fear of seeming to know less than the computer-savvy patient or a reluctance to learn new things. Alternatively, it could be the result of education selection and bias. Alternatively, there may be fear that technology will uncloak the mysticism that surrounds the medical prescribing process or make complicated disease management procedures more transparent.

One may recall that cardiologists were initially reluctant to accept the interpretation of the electrocardiogram by a computer. That fear was relatively short-lived and the machine-assisted interpretation now adds speed, objectivity, accuracy and reproducibility. Notably, the input and participation of undoubtedly sceptical (if not technophobic) practitioners accelerated the process of gaining acceptance. In clinical diabetes there seem to be few practitioners so inclined. If technophilia is a virtue of the cardiologist, then is technophobia a vice of the diabetes healthcare professional (diabetologist, internist, primary care provider, nurse, educator, dietician, nutritionist, exercise physiologist, psychologist, pharmacist, psychiatrist, etc)?

Some may argue that the failure to act is merely clinical inertia [9]. Where this is the case, information technology would seem to provide a way of relieving the inertia, by transferring the onus to act to the patient. If the patient fails to act, clinical inertia cannot be blamed. Nor can technophobia!

\section{What can information technology do in diabetes?}

Because a galaxy of complications can result from poor glycaemic control, contemporary diabetes management is becoming increasingly complex. With the addition of a team to support the physician and surround the patient, success in achieving metabolic control may require a concerted effort to undo or avoid fragmentation. Now, more than ever, the actual medical prescription may be the root cause of either brilliant treatment success or abysmal treatment failure.

For all concerned stakeholders, it is frustrating to know that an error of just \pm 1 unit of insulin or \pm 1 tablet of most of the oral agents can easily result in dangerous hypoglycaemia or unacceptable hyperglycaemia, certainly whenever the patient's blood glucose is in the mandated range. This treatment fact puts a heavy burden on the prescribers. Not only must the medication types and the actual doses be accurate, but also the details of the patient's education and training. Obviously, for many, this is a clinical dilemma, if not an impossibility.

\section{What has information technology done in other disciplines?}

In other disciplines, where similarly tight requirements develop, solutions of a technical nature have been recruited to great advantage. The process of landing interplanetary space probes on distant solar objects is one accomplishment that comes to mind. For that matter, even landing jumbo jets is now a hands-off process for pilots. Safety and the service life of airframes have both increased significantly through the use of computers to assist in smoothing the landing processes.

In other words, might it not be timely to encourage and support the introduction of similar information technologies into diabetes care? Without a push by the health providers for innovative technology, for both themselves and their patients, business will continue as usual. With just a minor push, great advances might occur. The squeaking wheel gets the oil.

\section{Never too late to act}

To join the debate, to participate in or to otherwise support the fledgling DCCT initiative, please contact the proponent at albisser@nidm.org_when nothing is ventured, nothing is gained. 
Duality of interest The author declares a duality of interest. His affiliation with the sponsoring institution of the DCCT initiative (BCMC Better Control Medical Computers) is as both an employee and a stakeholder.

\section{References}

1. DCCT Research Group (1993) The effect of intensive treatment of diabetes on the development and progression of long-term complications in insulin-dependent diabetes mellitus. $\mathrm{N}$ Engl $\mathrm{J}$ Med 329:977-986

2. UK Prospective Diabetes Study Group (1998) Intensive bloodglucose control with sulphonylureas or insulin compared with conventional treatment and risk of complications in patients with type 2 diabetes (UKPDS 33). Lancet 352:837-853

3. EDIC Research Group (1999) Epidemiology of Diabetes Interventions and Complications (EDIC): design, implementation, and preliminary results of a long-term follow-up of the Diabetes Control and Complications Trial cohort. Diabetes Care 22: 99-111

4. Lachin JM, Genuth S, Nathan DM, Zinman B, Rutledge BN; DCCT/EDIC Research Group (2008) Effect of glycemic exposure on the risk of microvascular complications in the diabetes control and complications trial-revisited. Diabetes 57:995-1001

5. The DCCT Research Group (1991) Epidemiology of severe hypoglycemia in the Diabetes Control and Complications Trial. Am J Med 90:450-459

6. Cryer PE (2008) Hypoglycemia: still the limiting factor in the glycemic management of diabetes. Endocr Pract 14:750-756

7. Albisser AM, Alejandro R, Sperlich M, Ricordi C (2009) Prescription checking device promises to resolve intractable hypoglycemia. J Diabetes Sci Technol (in press)

8. Albisser AM, Alejandro R, Sperlich M, Ricordi C (2009) Closing the circle of care with new firmware for diabetes: MyDiaBase+ RxChecker. J Diabetes Sci Technol (in press)

9. Phillips LS, Branch WT, Cook CB et al (2001) Clinical inertia. Ann Intern Med 135:825-834 